\section{PWE-178 POSITIVE OUTCOMES FROM A NUTRITION TEAM-LED RADIOLOGICALLY INSERTED GASTROSTOMY (RIG) SERVICE}

doi:10.1136/gutjnl-2013-304907.466

1," C Ryan-Fisher, 'N Burch, ${ }^{2} \mathrm{E}$ Trautner, ${ }^{2} \mathrm{~J}$ Colby, ${ }^{3} \mathrm{M}$ Dhillon, ${ }^{3} \mathrm{~J}$ Harding. 'Gastroenterology: ${ }^{2}$ Nutrition; ${ }^{3}$ Radiology, University Hospital Coventry and Warwickshire, Coventry, UK

Introduction Radiologically inserted gastrostomies (RIGs) are an important alternative method of enteral feeding when endoscopic technique is unfeasible. Current published data regarding results post-RIG insertion report varying success, with 30-day mortality ranging from $1-18 \% .^{1,2} \mathrm{~A}$ robust multidisciplinary assessment prior to RIG insertion is vital to ensure clinical suitability. We therefore have examined the case selection and clinical outcomes of our new RIG service.

Methods All patients who underwent RIG placement in our centre between February 2011 and November 2012 were identified. Retrospective analysis of the case notes established the complications post-RIG, the mortality data and which clinicians were involved with the pre-RIG assessment. Two clinicians also independently evaluated the clinical benefit of each RIG using criteria including weight gain, appropriate RIG dwell time and reliance on RIG delivered nutrition to complete proposed treatment (e.g. radiotherapy). Results 26 patients were identified (mean age 64 years, 54\% male). The indications for RIG were head and neck cancers (69\%), oesophageal malignancy (15\%) and neurological disorders (16\%). The Nutrition Team assessed $100 \%$ of patients prior to RIG and supported management of all patients post procedure. The mean time between referral and RIG was 6 days (range 2-15 days) and success rate for RIG placement was $96 \%$. The overall 30 day mortality was $4 \%$ ( 1 patient; unrelated to RIG). Early complications ( $<24$ hours) comprised 1 perforation with pneumoperitoneum. Late complications (2-30 days) included peristomal infection (15\%), stomal leakage $(12 \%)$ and bleeding from RIG site (4\%). Complications after 30 days included peristomal infection (8\%), inadvertent removal (8\%) and RIG tube blockage (8\%). Evaluation of clinical benefit concluded that $10 \%$ of patients did not benefit from their RIG: one patient with a perforation had a jejunostomy and one patient had leaking from the RIG site that was not utilised. For the remaining $90 \%$ of patients the RIG was judged a clinical success.

Conclusion Our data demonstrates an efficient and effective RIG service. Overall complication rates and 30-day mortality are low, reflecting RIG insertion as a safe alternative in those unsuitable for PEG. The assessment of the suitability for RIG by a multi-professional Nutrition Support Team and provision of ongoing support post insertion are key factors associated with a successful RIG service. Prospective data collection with a questionnaire could further evaluate patient experience.

Disclosure of Interest None Declared.

\section{REFERENCES}

1. Lowe AS, Laash HU, Stephenson S, et al. Clin Radiol 2012; 67:843-54.

2. Galaski A, Peng WW, Ellis M, et al. Can J Gastroenterol 2009; 23:109-14.

\section{PWE-179 NUTRITIONAL ASSESSMENT AND MANAGEMENT IN PATIENTS WITH DECOMPENSATED LIVER DISEASE}

doi:10.1136/gutjnl-2013-304907.467

1."D Rogers, 'V Gordon, ' J Stewart, ' $\mathrm{C}$ Tilley. ${ }^{1}$ University Hospitals of Leicester, Leicester, UK

Introduction Good nutrition is an effective intervention in decompensated liver disease ${ }^{1}$. Protein Energy Malnutrition (PEM) is found in $65-90 \%$ of patients with advanced liver disease and almost $100 \%$ of those awaiting transplant ${ }^{1}$. There is an association between nutritional status and mortality ${ }^{1}$ and PEM before transplantation leads to higher rates of post-transplant complications.
Reasons for PEM include ascites causing early satiety, alcohol use and malabsorption of fat soluble vitamins in cholestatic liver disease. Patients with cirrhosis have an altered metabolic response to starvation and overnight fasting can result in muscle depletion ${ }^{2}$. Daytime and evening snacks are therefore important, and hospital catering struggles to meet the nutritional needs of these patients. Clinicians should consider nutritional supplementation when admitting patients with cirrhosis.

Methods We audited 30 patients with decompensated liver disease who had been reviewed by a dietitian. Demographics, aetiology, prevalence of alcohol use and ascites were reviewed along with if the clerking or senior review doctor considered nutrition in their management plan. Dietetic reviews were audited to calculate the deficit in calorie and protein intake and if this was eliminated by the next review.

Results There were 18 male (60\%) and 12 female (40\%) patients with a mean age of 53 years (range 16-79). The most common aetiology was alcohol (25 patients) followed by Hepatitis C (2). $65 \%$ of those with alcoholic liver disease (ALD) were current drinkers (mean consumption 76 units/week, range 12-280). All non ALD aetiology were non-drinkers. Reason for admission was ascites (37\%), alcoholic hepatitis (30\%), encephalopathy (23\%) and variceal bleeding (10\%). 22/30 had ascites. No supplements or night time snacks were given and in only 3 was nutritional assessment planned.

$18(60 \%)$ were consuming under $500 \mathrm{kcal} /$ day and $15(50 \%)$ were consuming under $10 \mathrm{~g}$ of protein/day. 10 (33\%) had a deficit of over $2000 \mathrm{kcal} /$ day and $12(40 \%)$ had a protein deficit of over $80 \mathrm{~g} /$ day. By the second visit the deficit had been eliminated in 8 (26\%); 4 with supplements and 4 with NG feeding. 10 (34\%) had a reduced deficit, $9(30 \%)$ had no reduction and 3 patients had been discharged or died prior to review.

Conclusion We found a huge burden of PEM that is poorly recognised and acted upon. A Nutrition Care Pathway has been developed which should improve the nutritional care of these patients and encourage the use of supplements and snacks in addition to the hospital menu.

Disclosure of Interest None Declared.

\section{REFERENCES}

1. Henckel, AS, Buchman AL (2006) Nutritional support in chronic liver disease. Nature Clinical Practice Gastroenterology and Hepatology 3(4):202-9

2. The Nutrition and Hydration Digest: Improving Outcomes through Food and Beverage Services. British Dietetic Association (2012)

\section{PWE-180 THE ACIDIC POLYSACCHARIDE COMPONENT OF SOLUBLE PLANTAIN FIBRE INHIBITS THE ADHESION OF DIARRHOEA-ASSOCIATED PATHOGENS SALMONELLA, CLOSTRIDIUM DIFFICILE AND ENTEROTOXIGENIC ESCHERICHIA COLI TO INTESTINAL EPITHELIAL CELLS}

doi:10.1136/gutjnl-2013-304907.468

1."H L Simpson, 'B N Parsons, 'J M Rhodes, 'B J Campbell. 'Gastroenterology, UNIVERSITY OF LIVERPOOL, Liverpool, UK

Introduction Pathogen-related diarrhoea is a major problem worldwide, causing significant morbidity and mortality each year. Our recent studies have shown that soluble dietary fibre (non-starch polysaccharides, NSP), particularly those from plantain bananas (Musa spp.), can inhibit the adherence of diarrhoeal pathogens such as Salmonella, Clostridium difficile and enterotoxigenic Escherichia coli (ETEC) to the intestinal epithelium (J. Nutri. Biochem. 24:97-103). Our aim in this study was to elucidate specific polysaccharide components present in soluble plantain fibre that confer bioactivity to block diarrhoeal pathogen interaction with the gut epithelium. Methods Plantain NSP was separated into neutral and acidic component polysaccharides using $\mathrm{Q}$-Sepharose strong anion-exchange 
chromatography. Unbound neutral oligosaccharides were collected from the flow-through and $\mathrm{Q}$-Sepharose bound acidic polysaccharide fractions were eluted stepwise with $0.25,0.5$ and $1 \mathrm{M} \mathrm{NaCl}$. All fractions were desalted on PD MidiTrap G10 mini-columns. Purified fractions were assessed for inhibitory action on C. difficile 027A, ETEC C410 and S. Typhimurium adherence to human and porcine epithelial cell monolayers in the presence of plantain NSP and its fractions (30 min pre-treatment prior to infection), at MOI 100 for $90 \mathrm{~min}$ (for ETEC and Salmonella) or $120 \mathrm{~min}$ (for C. difficile). Adherent colony forming units (CFU) were enumerated by overnight growth on LB-agar.

Results As observed for plantain NSP, at $5 \mathrm{mg} / \mathrm{mL}$, a Q-Sepharosepurified acidic polysaccharide fraction of soluble plantain NSP also significantly blocked adhesion to human Caco2 colonocytes of gut pathogens $C$. difficile, by $52.3 \pm 7.3 \%$; ETEC, by $74.8 \pm 15.1$; and $S$. Typhimurium, by $92.1 \pm 2.91 \%$ (all $\mathrm{P}<0.001$ Kruskal-Wallis; $\mathrm{N}=2$ experiments, each with $\mathrm{n}=3$ replicates). Conversely, pre-treatment of $\mathrm{Caco} 2$ cells with the neutral polysaccharide fraction of plantain NSP showed little ability to reduce adhesion of $C$. difficile $(10.2 \%$ inhibition) and a reduced ability to inhibit interaction of Salmonella or ETEC (46.6\% and $39.1 \%$ inhibition respectively). Similalrly, acidic fractions of plantain NSP at $5 \mathrm{mg} / \mathrm{mL}$ also blocked adhesion of $\mathrm{S}$. Typhimurium to porcine enterocytic cell-line B10XI compared to untreated control ( $N=4, \mathrm{n}=4 ; P<0.01$ Kruskal-Wallis).

Conclusion Our findings indicate that the inhibitory activity of plantain NSP against diarrhoeal pathogen interaction with the host intestinal epithelium lies in its acidic (pectic) polysaccharide component. Disruption of bacterial-epithelial adherence to the intestinal mucosa by soluble plant fibres, acting as 'contrabiotics', may prove to be of therapeutic benefit.

Disclosure of Interest H. Simpson: None Declared, B. Parsons: None Declared, J. Rhodes Grant/Research Support from: Provexis plc (UK), Consultant for: A member of advisory boards for Atlantic, Proctor and Gamble, Falk, Conflict with: Lecture fees received from Abbott, Falk, Ferring, Glaxo Smith Kline, Proctor and Gamble, Schering Plough, Shire, Wyeth. With the University of Liverpool and Provexis UK, holds a patent for use of soluble plantain fibre as maintenance therapy for Crohn's disease plus a patent pending for its use in antibiotic associated diarrhoea, B. Campbell Grant/ Research Support from: Provexis plc. and Shire, Consultant for: Amgen Inc.

\section{PWE-181 THE DEVELOPMENT AND EVOLUTION OF NUTRITION SERVICE PROVISION IN THE NORTH EAST: 2003-2012}

doi:10.1136/gutjnl-2013-304907.469

1."J H Adam, 'N Thompson. 'Gastroenterology, Freeman Hospital, Newcastle Upon Tyne, Newcastle, UK

Introduction Malnutrition is well recognised as a treatable cause and result of ill-health. The recognition and management of malnutrition hinges upon the quality of organisation of nutrition services as well as the quality of the interventions themselves. Malnutrition is common amongst inpatients and decisions regarding management are often complex. The care of patients with complex nutritional needs can be optimised by combining the expertise of those from multiple allied disciplines. These principles are highlighted in the 2006 NICE guideline: Nutrition Support in Adults. This study analyses the progress made at an organisational level in providing nutrition services in hospitals across the North East of England, with reference to recommendations made by NICE.

Methods Nutrition service provision was evaluated by distributing questionnaires to all acute hospitals in the North East region in 2003, 2007 and 2012. Questionnaires were completed by a dietician from each hospital. The questionnaire addressed the provision of Nutrition Support Teams. We analysed the representation of team members from different disciplines, frequency of meetings and topics discussed.
We also sought qualitative and quantitative data regarding inpatient and outpatient use of Total Parenteral Nutrition (TPN), the use of malnutrition screening tools and access to obesity services.

Results Response rates were $92 \%$ in 2003 and $100 \%$ in 2007 and 2012. Over 9 years the percentage of hospitals with Nutrition Support Teams has increased from 19\% to 42\%. Each team discusses an increasing number of patients as well evaluating and developing their local policies and procedures. In line with NICE recommendations, $100 \%$ of acute trusts have a Nutrition Steering Committee in 2012, rising from $31 \%$ in 2003 and $94 \%$ in 2007 . The number of patients able to access inpatient TPN has increased across the region whilst the provision of home TPN has been concentrated towards larger, tertiary facilities. The use of standardised inpatient screening tools has increased from $80 \%$ in 2003 to $100 \%$ in 2012 . Screening of outpatients in acute hospitals has increased from $15 \%$ in 2003 to $33 \%$ in 2012 .

Conclusion Nutrition service provision has evolved beyond recognition over the last 9 years in the North East. Significant progress has been made in forming focused Nutrition Support Teams, expanding TPN delivery and detecting malnutrition, particularly among the inpatient population. Areas for improvement include the development and promotion of efficient and practical methods to screen outpatients for malnutrition. Ultimately, it is hoped that we can demonstrate our developments have had a significant and sustained impact upon patient care and outcomes.

Disclosure of Interest None Declared.

\section{REFERENCE}

1. Nutrition support in adults (CG32): 2006 NICE guideline

\section{PWE-182 MORTALITY AFTER PEG: REVIEW OF 259 PEG PROCEDURES AT A DISTRICT GENERAL HOSPITAL OVER A 7 YEAR PERIOD}

doi:10.1136/gutjnl-2013-304907.470

1,"K Thavanesan, 'K Barnett, 'S Harding, 'D Hartley, 'S Al-Shamma, 'S A Weaver, 'S D McLaughlin. 'Gastroenterology, Royal Bournemouth and Christchurch Hospital, Bournemouth, UK

Introduction Percutaneous Endoscopic Gastrostomy (PEG) is commonly performed in patients that require medium to long-term enteral feeding. In 2004 NCEPOD reviewed the 30day mortality and rate of complications following therapeutic endoscopy. They reported a $6 \% 30$ day mortality and made recommendations regarding changes in practise. Nationally there is little published data on the 1 year mortality following this procedure. In 2010 we introduced changes to our practise including: 1 . The review of all PEG referrals by a nutrition team, 2. Stringently avoiding PEG for 2 weeks following stroke, 3 . Avoiding PEG in patients with pneumonia or other inter-current illness.

Aim to review the 30day and 1 year mortality following PEG at our institution since the NCEPOD publication.

Methods A prospective endoscopy database is maintained at our institution (HICCS). We searched the database for primary PEG procedures during the period of Jan 2005 to September 2012 and reviewed electronic medical records to establish the overall 30 day and 1 year mortality, and also mortality by indication.

Results A total of 259 procedures were performed. Indications were: Stroke $n=130$ (50\%), Multiple sclerosis (MS) $n=28(10.85 \%)$, Motor neurone disease (MND) $\mathrm{n}=19(7.36 \%)$. Mortality data is given in table 1 .

Overall 30 day mortality by indication was: Stroke $10.8 \%, \mathrm{MND}$, $6.3 \%$, MS $12.5 \%$

1 year mortality: Stroke $31.27 \%$, MND 50.0\%, MS 30.7\%.

Conclusion Stroke is the commonest indication for PEG at our hospital. The mean annual 30day mortality following PEG at our hospital is above that reported by NCEPOD but has fallen considerably since 2010 suggesting that changes in our practise have been 